Konrad-Zuse-Zentrum

für Informationstechnik Berlin

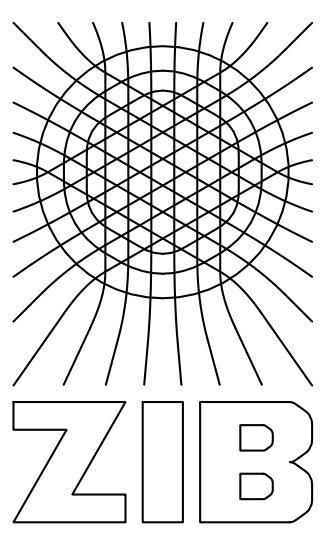

Takustraße 7 D-14195 Berlin-Dahlem

Akiyoshi Shioura, Ken'ICHIRo TANAKA

\title{
Polynomial-Time Algorithms \\ for Linear and Convex Optimization on Jump Systems
}




\title{
Polynomial-Time Algorithms for Linear and Convex Optimization on Jump Systems
}

\author{
Akiyoshi Shioura* and Ken'ichiro Tanaka ${ }^{\dagger}$
}

April, 2006

\begin{abstract}
The concept of jump system, introduced by Buchet and Cunningham (1995), is a set of integer points with a certain exchange property. In this paper, we discuss several linear and convex optimization problems on jump systems and show that these problems can be solved in polynomial time under the assumption that a membership oracle for a jump system is available. We firstly present a polynomial-time implementation of the greedy algorithm for the minimization of a linear function. We then consider the minimization of a separable-convex function on a jump system, and propose the first polynomial-time algorithm for this problem. The algorithm is based on the domain reduction approach developed in Shioura (1998). We finally consider the concept of M-convex functions on constant-parity jump systems which has been recently proposed by Murota (2006). It is shown that the minimization of an M-convex function can be solved in polynomial time by the domain reduction approach.
\end{abstract}

\section{Introduction}

The concept of jump system, introduced by Buchet and Cunningham [7], is a set of integer points with a certain exchange property (to be described in Section 2); see also $[9,13,18]$. It is a generalization of a matroid $[17,24,27]$, a delta-matroid $[6,8]$, and the base polyhedron of an integral polymatroid (or a submodular system) $[11,24,27]$. Jump systems have various examples (see $[7,9,13,18])$; in particular, the degree sequences of subgraphs of a graph is a fundamental example of jump systems. In this paper, we investigate the following linear and convex optimization problems on jump systems:

\footnotetext{
*Konrad-Zuse-Zentrum für Informationstechnik Berlin Takustraße 7, 14195 Berlin, Germany. On leave from Graduate School of Information Sciences, Tohoku University, Sendai 980-8579, Japan. shioura@dais.is.tohoku.ac.jp

'Graduate School of Information Science and Technology, University of Tokyo, Tokyo 1138656, Japan. kenitiro@misojiro.t.u-tokyo.ac.jp.
} 
(LFMin) minimization of a linear function on a jump system,

(ScFMin) minimization of a separable-convex function

(McFMin) minimization of an M-convex function

on a jump system,

on a constant-parity jump system.

The main aim of this paper is to show that these problems can be solved in polynomial time under the assumption that a membership oracle for a jump system is available.

\subsection{Linear Optimization on Jump Systems}

We discuss the greedy algorithm for the problem (LFMin) in Section 3. It is shown [7] (see also $[9,13,18]$ ) that the problem (LFMin) can be solved by a greedy algorithm. The greedy algorithm finds an optimal solution by iteratively calling a procedure for solving a problem of minimizing (or maximizing) some component of a vector on a jump system. However, the time-complexity of the greedy algorithm is not analyzed in $[7,9,13,18]$, and it is not known so far whether the greedy algorithm runs in polynomial time or not, provided a membership oracle for a jump system is available.

In this paper, we show that the greedy algorithm runs in polynomial time. In particular, we present an implementation of the procedure mentioned above and prove that the procedure runs in polynomial time.

\subsection{Convex Optimization on Jump Systems}

In Section 4, we consider two convex optimization problems (ScFMin) and (McFMin), and propose polynomial-time algorithms for these problems.

We first consider the problem (ScFMin) in Section 4.1. A canonical example of this problem arises from the minimization of a separable convex function on the degree sequences of an undirected graph; a related problem called the minsquare factor problem is discussed in $[4,5]$. The problem (ScFMin) is studied in [3], where a local criterion for optimality as well as a greedy algorithm is given. Although it is shown that the greedy algorithm runs in pseudo-polynomial time, it is not known so far whether the problem (ScFMin) can be solved in polynomial time.

On the other hand, some special cases of (ScFMin) can be solved in polynomial time. One of such cases is the problem on integral base polyhedra, which is extensively discussed and several efficient algorithms have been proposed $[14,15,20]$. Another well-solved case is the problem on integral bisubmodular polyhedra, to which Fujishige [10] applied a min-max theorem for bisubmodular polyhedra and developed a polynomial-time algorithm.

In this paper, we present the first polynomial-time algorithm for the problem (ScFMin). Our algorithm is based on the domain reduction approach [25], which is originally developed for the minimization of a class of discrete convex functions called M-convex functions on base polyhedra [21]. One of the key properties to the domain reduction approach is the "minimizer cut property," which states that a given feasible vector can be easily separated from an optimal solution. We show that the minimizer cut property indeed holds for the problem (ScFMin). By repeatedly applying the minimizer cut property to appropriately 
chosen feasible vectors, we show that the algorithm finds an optimal solution in polynomial time.

We then discuss in Section 4.2 an application of our algorithm to the problem of finding least weakly sub- and supermajorized elements. The concept of (weak) majorization plays a fundamental role in fair resource allocation and related problems (see [19]), and it is shown that any jump system has least weakly suband supermajorized elements [1]. By using our algorithm as well as the result in [1], we show that the problem of finding least weakly sub- and supermajorized elements in jump systems can be solved in polynomial time.

We finally consider the problem (McFMin) in Section 4.3. The concept of M-convex functions is originally introduced by Murota [21] for functions defined on base polyhedra, and recently generalized for functions defined on constantparity jump systems [22], with a view to providing a general framework for the minsquare factor problem on undirected graphs [4, 5]. Examples of Mconvex functions on constant-parity jump systems include a nonseparable convex function arising from the minimum weight perfect $b$-matching problem as well as a separable-convex function on the degree sequences of an undirected graphs (see Section 2). Fundamental properties of M-convex functions on constant-parity jump systems are investigated in $[16,22,23]$, where it is shown that a local optimality criterion guarantees global optimality and that a greedy algorithm solves the problem (McFMin) in pseudo-polynomial time.

In this paper, we present the first polynomial-time algorithm for the problem (McFMin), which is also based on the domain reduction approach. In fact, the minimizer cut property for (McFMin) is already shown in [23]. By using this fact, we show that a variant of the polynomial-time algorithm for (ScFMin) finds an optimal solution of (McFMin) in polynomial time.

\section{Preliminaries on Jump Systems}

Let $V$ be a nonempty finite set, and put $n=|V|$. We denote the set of reals and integers by $\mathbb{R}$ and by $\mathbb{Z}$, respectively. Also, we denote by $\mathbb{Z}_{+}$the set of nonnegative integers. For $x=(x(v) \mid v \in V) \in \mathbb{R}^{V}$, we define

$x(Y)=\sum_{v \in Y} x(v)(Y \subseteq V), \quad\|x\|_{1}=\sum_{v \in V}|x(v)|, \quad \operatorname{supp}(x)=\{v \in V \mid x(v) \neq 0\}$.

We denote by $\mathbf{0}$ the zero vector in $\mathbb{R}^{V}$. For $u \in V$ we denote by $\chi_{u}$ the characteristic vector of $u$, with $\chi_{u}(u)=1$ and $\chi_{u}(v)=0$ for $v \neq u$. We denote by $N_{1}$ the set of all integral vectors $x$ with $\|x\|_{1}=1$, i.e., $N_{1}=\left\{+\chi_{v},-\chi_{v} \mid v \in V\right\}$. For a nonempty finite set $S \subseteq \mathbb{Z}^{V}$, we define the size $\Phi(S)$ of $S$ by

$$
\Phi(S)=\max _{x, y \in S}\|x-y\|_{1}\left(=\max _{v \in V}\left\{\max _{y \in S} y(v)-\min _{y \in S} y(v)\right\}\right) .
$$

For $x, y \in \mathbb{Z}^{V}$, a vector $s \in N_{1}$ is said to be an $(x, y)$-increment if it satisfies $\|(x+s)-y\|_{1}=\|x-y\|_{1}-1$. We denote by $\operatorname{inc}(x, y)$ the set of all $(x, y)$ increments. A nonempty set $J \subseteq \mathbb{Z}^{V}$ is said to be a jump system if it satisfies the following exchange axiom:

$\left(\mathbf{J}_{-\mathbf{E X C}}\right)$ For any $x, y \in J$ and for any $s \in \operatorname{inc}(x, y)$, if $x+s \notin J$ then there exists $t \in \operatorname{inc}(x+s, y)$ such that $x+s+t \in J$. 
A set $J \subseteq \mathbb{Z}^{V}$ is said to be a constant-parity system if $x(V)-y(V)$ is even for any $x, y \in J$.

We here mention some elementary operations which preserve the property $\left(\mathrm{J}-\mathrm{EXC}_{0}\right)$. Jump systems are closed under reflection.

Proposition $2.1([7])$. Let $J \subseteq \mathbb{Z}^{V}$ be a jump system and $u \in V$. Then, the set

$$
J^{u}=\left\{y \in \mathbb{Z}^{V} \mid \exists x \in J \text { s.t. } y(u)=-x(u), y(v)=x(v)(v \in V \backslash\{u\})\right\}
$$

is a jump system.

For any vectors $a, b \in \mathbb{Z}^{V}$ with $a \leq b$, we define a $b o x[a, b]$ by

$$
[a, b]=\left\{x \in \mathbb{Z}^{V} \mid a(v) \leq x(v) \leq b(v)(v \in V)\right\} .
$$

Proposition 2.2 (cf. [7]). Let $J \subseteq \mathbb{Z}^{V}$ be a jump system, and $a, b \in \mathbb{Z}^{V}$ be vectors with $a \leq b$. Then, $J \cap[a, b]$ is a jump system if it is nonempty

A univariate function $\varphi: \mathbb{Z} \rightarrow \mathbb{R}$ is convex if it satisfies

$$
2 \varphi(\alpha) \leq \varphi(\alpha-1)+\varphi(\alpha+1) \quad(\forall \alpha \in \mathbb{Z}) .
$$

A function $f: \mathbb{Z}^{V} \rightarrow \mathbb{R}$ is said to be separable-convex if it is a function of the form $f(x)=\sum_{v \in V} f_{v}(x(v))$ with univariate convex functions $f_{v}: \mathbb{Z} \rightarrow \mathbb{R}(v \in V)$. The sum of squares $f(x)=\sum_{v \in V}(x(v))^{2}$ is a special case of a separable-convex function.

Let $J \subseteq \mathbb{Z}^{V}$ be a constant-parity jump system. A function $f: J \rightarrow \mathbb{R}$ is said to be $M$-convex if it satisfies the following property:

For any $x, y \in J$ and for any $s \in \operatorname{inc}(x, y)$, there exists $t \in \operatorname{inc}(x+s, y)$ such that $x+s+t \in J, y-s-t \in J$, and

$$
f(x)+f(y) \geq f(x+s+t)+f(y-s-t) .
$$

We note that the exchange axiom:

(J-EXC) For any $x, y \in J$ and for any $s \in \operatorname{inc}(x, y)$, there exists $t \in \operatorname{inc}(x+s, y)$ such that $x+s+t \in J$ and $y-s-t \in J$

characterizes a constant-parity jump system, a fact credited to J. Geelen (see [22] for a proof).

Proposition 2.3. A nonempty set $J \subseteq \mathbb{Z}^{V}$ is a constant-parity jump system if and only if it satisfies (J-EXC).

Examples of jump systems and M-convex functions follow.

Example 2.4. Let $G=(V, E)$ be an undirected graph that may contain loops and parallel edges. For a subgraph $H=(V, F)$ of $G$, denote its degree sequence by $\operatorname{deg}_{H}=\sum\left\{\chi_{u}+\chi_{v} \mid(u, v) \in F\right\} \in \mathbb{Z}^{V}$. It is well known $[7,9,13,18]$ that

$$
J=\left\{\operatorname{deg}_{H} \mid H \text { is a subgraph of } G\right\}
$$


forms a constant-parity jump system, called the degree system of $G$. Minimization of a separable-convex function on the degree system $J$ has been investigated in $[4,5]$.

Given a edge weight function $w: E \rightarrow \mathbb{R}$, we define a function $f: J \rightarrow \mathbb{R}$ by

$$
f(x)=\min \left\{\sum_{e \in F} w(e) \mid H=(V, F) \text { is a subgraph of } G \text { with } \operatorname{deg}_{H}=x\right\},
$$

which represents the minimum weight of a subgraph with degree sequence $x$. Then, $f$ is an M-convex function on a constant-parity jump system [22].

Example 2.5 ([23]). Let $G=(V, E)$ be an undirected graph that may have loops, but no parallel edges. Let $w: E \rightarrow \mathbb{R}$ be an edge weight function, and $c: E \rightarrow \mathbb{Z}_{+}$be an edge capacity function. We define $J \subseteq \mathbb{Z}^{V}$ as the set of vectors $x \in \mathbb{Z}^{V}$ such that a $c$-capacitated perfect $x$-matching exists in $G$, i.e., such that there exists $\lambda \in \mathbb{Z}^{E}$ satisfying

$\sum\{\lambda(e) \mid$ edge $e$ is incident to $v\}=x(v)(\forall v \in V), \quad 0 \leq \lambda(e) \leq c(e)(\forall e \in E)$.

Then, $J$ is a constant-parity jump system.

We then define a function $f: J \rightarrow \mathbb{R}$ as the minimum weight of a $c$ capacitated perfect $x$-matching, i.e.,

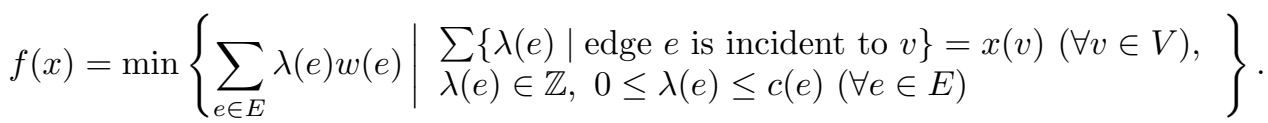

Then, $f$ is an M-convex function on a constant-parity jump system. Moreover, the function $\tilde{f}: J \rightarrow \mathbb{R}$ given as

$$
\tilde{f}(x)=f(x)+\sum_{v \in V} f_{v}(x(v)),
$$

where $f_{v}: \mathbb{Z} \rightarrow \mathbb{R}(v \in V)$ is a family of univariate convex functions, is also M-convex.

\section{Polynomiality of the Greedy Algorithm for Linear Optimization on Jump Systems}

In this section, we consider the problem of minimizing a linear function on a jump system:

(LFMin) Minimize $w^{T} x \quad$ subject to $\quad x \in J$,

where $w \in \mathbb{R}^{V}$ and $J$ is a finite jump system. We show that the greedy algorithm for the problem (LFMin) runs in polynomial time. We assume that a membership oracle for the jump system $J$ is available.

\subsection{Greedy Algorithm}

It is shown $[7,18]$ that the problem (LFMin) can be solved by the following greedy algorithm: 
Algorithm Greedy

Step 0: Let $x_{0}$ be any vector in $J$. Put $J_{0}=J$. Order the elements in $V=$ $\left\{v_{1}, v_{2}, \ldots, v_{n}\right\}$ so that

$$
\left|w\left(v_{1}\right)\right| \geq \cdots \geq\left|w\left(v_{k}\right)\right|>\left|w\left(v_{k+1}\right)\right|=\cdots=\left|w\left(v_{n}\right)\right|=0 .
$$

Step 1: For $i=1,2, \ldots, k$, do the following:

Step 1-1: Compute the value $\alpha_{i} \in \mathbb{Z}$ given by

$$
\alpha_{i}= \begin{cases}\min \left\{y\left(v_{i}\right) \mid x \in J_{i-1}\right\} & \left(\text { if } w_{i}>0\right), \\ \max \left\{y\left(v_{i}\right) \mid x \in J_{i-1}\right\} & \left(\text { if } w_{i}<0\right) .\end{cases}
$$

Step 1-2: Let $x_{i}$ be any vector in $J_{i-1}$ with $x_{i}\left(v_{i}\right)=\alpha_{i}$.

Step 2: Output $x_{k}$.

Put $J_{i}=\left\{y \in J_{i-1} \mid y\left(v_{i}\right)=\alpha_{i}\right\}$.

Theorem $3.1([7,18])$. The algorithm GREEDY outputs an optimal solution of (LFMin).

We show that the algorithm GREEDY runs in polynomial time.

Theorem 3.2. The algorithm GREEDY finds an optimal solution of (LFMin) in $\mathrm{O}\left(n^{2} \log \Phi(J)\right)$ time, provided a vector in $J$ is given.

Proof. The most time-consuming part is the computation of $\alpha_{i}$ in Step 1-1, which can be done in $\mathrm{O}(n \log \Phi(J))$ time by using the vector $x_{i-1}$, as shown later in Section 3.2. Hence, the algorithm GrEedy runs in $\mathrm{O}\left(n^{2} \log \Phi(J)\right)$ time. time.

In the next section we explain in details how to compute $\alpha_{i}$ in $\mathrm{O}(n \log \Phi(J))$

\subsection{Computation of Upper and Lower Bounds of Jump Systems}

We present two procedures to compute the values $\max \{y(u) \mid y \in J\}$ and $\min \{y(u) \mid y \in J\}$ in polynomial time for a finite jump system $J \subseteq \mathbb{Z}^{V}$ and an element $u \in V$.

Procedure Upper_Bound $(J, u)$

Step 0: Let $x:=x_{0}$ be an initial vector in $J$.

Step 1: Put $x:=x+\bar{\alpha} \chi_{u}$, where $\bar{\alpha}=\max \left\{\alpha \in \mathbb{Z}_{+} \mid x+\alpha \chi_{u} \in J\right\}$.

Step 2: For each $v \in V \backslash\{u\}$, do the following:

Step 2-1: Put $x:=x+\bar{\beta}_{v}\left(\chi_{u}+\chi_{v}\right)$, where

$$
\bar{\beta}_{v}=\max \left\{\beta \in \mathbb{Z}_{+} \mid x+\beta\left(\chi_{u}+\chi_{v}\right) \in J\right\} .
$$

Step 2-2: Put $x:=x+\bar{\gamma}_{v}\left(\chi_{u}-\chi_{v}\right)$, where

$$
\bar{\gamma}_{v}=\max \left\{\gamma \in \mathbb{Z}_{+} \mid x+\gamma\left(\chi_{u}-\chi_{v}\right) \in J\right\} .
$$

Step 3: Output $x$.

Procedure Lower_Bound $(J, u)$

Step 0: Let $x:=x_{0}$ be an initial vector in $J$. 
Step 1: Put $x:=x-\bar{\alpha} \chi_{u}$, where $\bar{\alpha}=\max \left\{\alpha \in \mathbb{Z}_{+} \mid x-\alpha \chi_{u} \in J\right\}$.

Step 2: For each $v \in V \backslash\{u\}$, do the following:

Step 2-1: Put $x:=x+\bar{\beta}_{v}\left(-\chi_{u}+\chi_{v}\right)$, where

$$
\bar{\beta}_{v}=\max \left\{\beta \in \mathbb{Z}_{+} \mid x+\beta\left(-\chi_{u}+\chi_{v}\right) \in J\right\} .
$$

Step 2-2: Put $x:=x+\bar{\gamma}_{v}\left(-\chi_{u}-\chi_{v}\right)$, where

$$
\bar{\gamma}_{v}=\max \left\{\gamma \in \mathbb{Z}_{+} \mid x+\gamma\left(-\chi_{u}-\chi_{v}\right) \in J\right\} .
$$

Step 3: Output $x$.

Theorem 3.3. For a finite jump system $J$ and $u \in V$, the procedure Up$\operatorname{PER} \_B O u n d(J, u)$ (resp., LOWER_Bound $(J, u)$ ) finds a vector $x \in J$ satisfying $x(u)=\max \{y(u) \mid y \in J\}$ (resp., $x(u)=\min \{y(u) \mid y \in J\})$ in $\mathrm{O}(n \log \Phi(J))$ time, provided a vector $x_{0} \in J$ is given.

The proof of Theorem 3.3 is given below in Sections 3.2.1 and 3.2.2.

Corollary 3.4. Suppose that $J$ is a jump system given as the intersection $J=$ $\tilde{J} \cap[a, b]$ of another jump system $\tilde{J}$ and a box $[a, b]$, and that a membership oracle for $\tilde{J}$ is available. For any $u \in V$, we can find vectors $x, x^{\prime} \in J$ with $x(u)=\max \{y(u) \mid y \in J\}$ and $x^{\prime}(u)=\min \{y(u) \mid y \in J\}$ in $\mathrm{O}(n \log \Phi(J))$ time, provided a vector in $J$ is given.

Proof. Although it takes $\mathrm{O}(n)$ time to check whether a given vector is contained in $\tilde{J} \cap[a, b]$, we can implement the procedures $\operatorname{UpPER}$ Bound $(J, u)$ and LOWER_Bound $(J, u)$ so that they run in $\mathrm{O}(n \log \Phi(J))$ time.

When the procedures need to check whether $x \in \tilde{J} \cap[a, b]$, the vector $x$ is of the form $x=y+\alpha\left(\chi_{u} \pm \chi_{v}\right)$ with $y \in \tilde{J} \cap[a, b], \alpha \in \mathbb{Z}_{+}$, and $v \in V$. Hence, we have $x \in \tilde{J} \cap[a, b]$ if and only if $x \in \tilde{J}, a(u) \leq x(u) \leq b(u)$, and $a(v) \leq x(v) \leq b(v)$, which can be checked in constant time. This shows that the procedures run in $\mathrm{O}(n \log \Phi(J))$ time for the jump system $J=\tilde{J} \cap[a, b]$ as well.

\subsubsection{Validity}

We show the validity of the procedure $\operatorname{Upper} \operatorname{Bound}(J, u)$. The validity of LOWER_Bound $(J, u)$ can be shown similarly and therefore omitted.

Lemma 3.5. Let $x \in J$ and $u \in V$. Suppose that $x+\chi_{u}+t \notin J$ holds for all $t \in\left(N_{1} \cup\{\mathbf{0}\}\right) \backslash\left\{-\chi_{u}\right\}$. Then, we have $x(u)=\max \{y(u) \mid y \in J\}$.

Proof. Assume, to the contrary, that there exists some $x^{\prime} \in J$ with $x^{\prime}(u)>x(u)$. Since $x+\chi_{u} \notin J$ by assumption, (J-EXC $)_{0}$ implies that there exists some $t \in$ $\operatorname{inc}\left(x+\chi_{u}, x^{\prime}\right)$ such that $x+\chi_{u}+t \in J$, a contradiction since $t \in N_{1} \backslash\left\{-\chi_{u}\right\}$.

Lemma 3.6. Let $u \in V$, and $x, y \in J$ be vectors such that

$$
y(u)-x(u) \geq \sum_{v \in V \backslash\{u\}}|x(v)-y(v)|+1 .
$$

Then, we have $\left\{x+\chi_{u}, x+2 \chi_{u}\right\} \cap J \neq \emptyset$. 
Proof. We prove the claim by induction on the value $\|x-y\|_{1}$.

We first consider the case where $x(v)=y(v)$ for all $v \in V \backslash\{u\}$, which contains the base case where $\|x-y\|_{1}=1$. Then, $\left(\mathrm{J}_{-} \mathrm{EXC}_{0}\right)$ for $x$ and $y$ implies $\left\{x+\chi_{u}, x+2 \chi_{u}\right\} \cap J \neq \emptyset$.

We then assume that $x(w) \neq y(w)$ for some $w \in V \backslash\{u\}$, where it may be assumed that $x(w)<y(w)$. Since $-\chi_{w} \in \operatorname{inc}(y, x),\left(\mathrm{J}_{-} \mathrm{EXC}_{0}\right)$ for $y$ and $x$ implies that there exists $t \in \operatorname{inc}\left(y-\chi_{w}, x\right) \cup\{\mathbf{0}\}$ such that $y^{\prime}=y-\chi_{w}+t \in J$. The vector $y^{\prime}$ satisfies

$y^{\prime}(u)-x(u) \geq y(u)-x(u)-1 \geq \sum_{v \in V \backslash\{u\}}|x(v)-y(v)| \geq \sum_{v \in V \backslash\{u\}}\left|x(v)-y^{\prime}(v)\right|+1$ and $\left\|y^{\prime}-x\right\|_{1}<\|y-x\|_{1}$. Hence, the induction hypothesis implies $\left\{x+\chi_{u}, x+\right.$ $\left.2 \chi_{u}\right\} \cap J \neq \emptyset$.

Lemma 3.7. Let $u \in V$, and $x, y \in J$ be vectors such that

$$
y(u)-x(u) \geq \sum_{v \in V \backslash\{u\}}|x(v)-y(v)| .
$$

If $\left\{x+\chi_{u}, x+2 \chi_{u}\right\} \cap J=\emptyset$, then $\left\{y+\chi_{u}, y+2 \chi_{u}\right\} \cap J=\emptyset$.

Proof. Suppose, to the contrary, that $\left\{y+\chi_{u}, y+2 \chi_{u}\right\} \cap J \neq \emptyset$ and let $y^{\prime} \in$ $\left\{y+\chi_{u}, y+2 \chi_{u}\right\} \cap J$. Then, we have $y^{\prime}(u)-x(u) \geq \sum_{v \in V \backslash\{u\}}\left|x(v)-y^{\prime}(v)\right|+1$, and therefore $\left\{x+\chi_{u}, x+2 \chi_{u}\right\} \cap J \neq \emptyset$ by Lemma 3.6, a contradiction.

Lemma 3.8. Let $u, w \in V$ be distinct elements, and $x, y \in J$ be vectors such that

$$
y(u)-x(u) \geq \sum_{v \in V \backslash\{u\}}|x(v)-y(v)|, \quad|x(w)-y(w)| \geq 1 .
$$

(i) If $x(w)<y(w)$, then $\left\{x+\chi_{u}, x+2 \chi_{u}, x+\chi_{u}+\chi_{w}\right\} \cap J \neq \emptyset$.

(ii) If $x(w)>y(w)$, then $\left\{x+\chi_{u}, x+2 \chi_{u}, x+\chi_{u}-\chi_{w}\right\} \cap J \neq \emptyset$.

Proof. We prove (i) by induction on the value $\|x-y\|_{1}$. The claim (ii) can be shown similarly.

We first consider the case where $x(v)=y(v)$ holds for all $v \in V \backslash\{u, w\}$, which contains the base case where $\|x-y\|_{1}=2$. Then, $y=x+\alpha \chi_{u}+\beta \chi_{w}$ for some positive integers $\alpha$ and $\beta$ with $\alpha \geq \beta$. Since $+\chi_{u} \in \operatorname{inc}(x, y)$, (J-EXC $\left.\mathrm{E}_{0}\right)$ for $x$ and $y$ implies $\left\{x+\chi_{u}, x+2 \chi_{u}, x+\chi_{u}+\chi_{w}\right\} \cap J \neq \emptyset$.

We then assume $x\left(v^{\prime}\right) \neq y\left(v^{\prime}\right)$ for some $v^{\prime} \in V \backslash\{u, w\}$, where we may assume $x\left(v^{\prime}\right)<y\left(v^{\prime}\right)$. Since $-\chi_{v^{\prime}} \in \operatorname{inc}(y, x),\left(J-\mathrm{EXC}_{0}\right)$ for $y$ and $x$ implies $y^{\prime}=y-\chi_{v^{\prime}}+t \in J$ for $t \in \operatorname{inc}\left(y-\chi_{v^{\prime}}, x\right) \cup\{\mathbf{0}\}$.

[Case 1: $t \neq-\chi_{u}$ ] $\quad y^{\prime}$ satisfies

$$
y^{\prime}(u)-x(u)=y(u)-x(u) \geq \sum_{v \in V \backslash\{u\}}|x(v)-y(v)| \geq \sum_{v \in V \backslash\{u\}}\left|x(v)-y^{\prime}(v)\right|+1 .
$$

Hence, we have $\left\{x+\chi_{u}, x+2 \chi_{u}\right\} \cap J \neq \emptyset$ by Lemma 3.6.

[Case 2: $\left.t=-\chi_{u}\right] \quad$ We have

$y^{\prime}(u)-x(u)=y(u)-x(u)-1 \geq \sum_{v \in V \backslash\{u\}}|x(v)-y(v)|-1=\sum_{v \in V \backslash\{u\}}\left|x(v)-y^{\prime}(v)\right|$,

and $y^{\prime}(w)=y(w)>x(w)$. Since $\left\|y^{\prime}-x\right\|_{1}=\|y-x\|_{1}-2$, the induction hypothesis implies $\left\{x+\chi_{u}, x+2 \chi_{u}, x+\chi_{u}+\chi_{w}\right\} \cap J \neq \emptyset$. 
Lemma 3.9. Let $u, w \in V$ be distinct elements, and $x, y \in J$ be vectors such that

$$
y(u)-x(u) \geq \sum_{v \in V \backslash\{u\}}|x(v)-y(v)| .
$$

Then, we have the following:

(i) If $\left\{x+\chi_{u}, x+2 \chi_{u}, x+\chi_{u}+\chi_{w}\right\} \cap J=\emptyset$, then $y+\chi_{u}+\chi_{w} \notin J$.

(ii) If $\left\{x+\chi_{u}, x+2 \chi_{u}, x+\chi_{u}-\chi_{w}\right\} \cap J=\emptyset$, then $y+\chi_{u}-\chi_{w} \notin J$.

Proof. We prove (i) only. Assume, to the contrary, that $y^{\prime}=y+\chi_{u}+\chi_{w} \in J$.

We first consider the case where $y(w) \geq x(w)$. Then,

$y^{\prime}(u)-x(u)=y(u)-x(u)+1 \geq \sum_{v \in V \backslash\{u\}}|x(v)-y(v)|+1=\sum_{v \in V \backslash\{u\}}\left|x(v)-y^{\prime}(v)\right|$

and $y^{\prime}(w)-x(w)=y(w)-x(w)+1>0$. Hence, Lemma 3.8 implies $\left\{x+\chi_{u}, x+\right.$ $\left.2 \chi_{u}, x+\chi_{u}+\chi_{w}\right\} \cap J \neq \emptyset$, a contradiction.

We then consider the case where $y(w)<x(w)$. Then,

$y^{\prime}(u)-x(u)=y(u)-x(u)+1 \geq \sum_{v \in V \backslash\{u\}}|x(v)-y(v)|+1=\sum_{v \in V \backslash\{u\}}\left|x(v)-y^{\prime}(v)\right|+2$.

Hence, Lemma 3.6 implies $\left\{x+\chi_{u}, x+2 \chi_{u}\right\} \cap J \neq \emptyset$, a contradiction.

Lemma 3.10. The procedure $\operatorname{UPPER} \operatorname{Bound}(J, u)$ finds a vector $x \in J$ satisfying $x(u)=\max \{y(u) \mid y \in J\}$.

Proof. By the definition of $\bar{\alpha}$, we have $\left\{x+\chi_{u}, x+2 \chi_{u}\right\} \cap J=\emptyset$ just immediately after Step 1. Therefore, $\left\{x+\chi_{u}, x+2 \chi_{u}\right\} \cap J=\emptyset$ holds during the iterations in Step 2 by Lemma 3.7. Similarly, we have $x+\chi_{u}+\chi_{w} \notin J$ (resp., $x+\chi_{u}-\chi_{w} \notin J$ ) just immediately after Step 2-1 (resp., Step 2-2) with $v=w$ is performed, and therefore $x+\chi_{u}+\chi_{w} \notin J$ (resp., $x+\chi_{u}-\chi_{w} \notin J$ ) holds in the following iterations in Step 2 by Lemma 3.9. At the end of procedure, the vector $x$ satisfies $x+\chi_{u}+t \notin J$ for all $t \in\left(N_{1} \cup\{\mathbf{0}\}\right) \backslash\left\{-\chi_{u}\right\}$. Hence, Lemma 3.5 implies $x(u)=\max \{y(u) \mid y \in J\}$.

\subsubsection{Time Complexity}

We then analyze the time complexity of the procedure $\operatorname{Upper} \operatorname{Bound}(J, u)$. The analysis of $\operatorname{LOWER\_ Bound}(J, u)$ is similar and therefore omitted.

Lemma 3.11. Let $x \in J$ and $u \in V$, and put $\bar{\alpha}=\max \left\{\alpha \in \mathbb{Z}_{+} \mid x+\alpha \chi_{u} \in J\right\}$. Then, we have $\left\{x+\alpha \chi_{u}, x+(\alpha+1) \chi_{u}\right\} \cap J \neq \emptyset$ for any $\alpha \in \mathbb{Z}$ with $0 \leq \alpha<\bar{\alpha}$.

Proof. The claim follows immediately from $\left(\mathrm{J}_{-} \mathrm{EXC}_{0}\right)$.

Lemma 3.12. Let $x \in J$, and $u, w \in V$ be distinct elements. Suppose that $\left\{x+\chi_{u}, x+2 \chi_{u}\right\} \cap J=\emptyset$.

(i) Let $\bar{\beta}_{w}=\max \left\{\beta \in \mathbb{Z}_{+} \mid x+\beta\left(\chi_{u}+\chi_{w}\right) \in J\right\}$. Then, $x+\beta\left(\chi_{u}+\chi_{w}\right) \in J$ for all $\beta \in \mathbb{Z}$ with $0 \leq \beta \leq \bar{\beta}_{w}$.

(ii) Let $\bar{\gamma}_{w}=\max \left\{\gamma \in \mathbb{Z}_{+} \mid x+\gamma\left(\chi_{u}-\chi_{w}\right) \in J\right\}$. Then, $x+\gamma\left(\chi_{u}-\chi_{w}\right) \in J$ for all $\gamma \in \mathbb{Z}$ with $0 \leq \gamma \leq \bar{\gamma}_{w}$. 
Proof. We prove (i) only. It suffices to show that for any positive integer $\beta$ with $x+\beta\left(\chi_{u}+\chi_{w}\right) \in J$, it holds that $x+(\beta-1)\left(\chi_{u}+\chi_{w}\right) \in J$. By (J$\left.\mathrm{EXC}_{0}\right)$ applied to $y=x+\beta\left(\chi_{u}+\chi_{w}\right)$ and $x$, we have $y-\chi_{w}+t \in J$ for some $t \in\left\{\mathbf{0},-\chi_{u},-\chi_{w}\right\}$. Since $\left\{x+\chi_{u}, x+2 \chi_{u}\right\} \cap J=\emptyset$, it follows from Lemma 3.6 that $\left\{y-\chi_{w}, y-2 \chi_{w}\right\} \cap J=\emptyset$. Therefore, we have $y-\chi_{w}-\chi_{u}=$ $x+(\beta-1)\left(\chi_{u}+\chi_{w}\right) \in J$.

Lemma 3.13. For any $u \in V$, the procedure $\operatorname{UpPER} \_\operatorname{Bound}(J, u)$ runs in $\mathrm{O}(n \log \Phi(J))$ time, provided a vector $x_{0} \in J$ is given.

Proof. By Lemma 3.11, the value $\bar{\alpha}$ in Step 1 can be computed in $\mathrm{O}(\log \Phi(J))$ time by a variant of binary search. Similarly, we can compute $\bar{\beta}_{v}$ and $\bar{\gamma}_{v}(v \in$ $V \backslash\{u\})$ by binary search in $\mathrm{O}(\log \Phi(J))$ time by Lemma 3.12 . Hence, the claim follows.

This concludes the proof of Theorem 3.3.

\section{Polynomial-Time Algorithms for Convex Op- timization on Jump Systems}

In this section, we consider the following two convex optimization problems on jump systems. The first problem is the minimization of a separable-convex function on a jump system:

$$
\text { (ScFMin) Minimize } f(x) \equiv \sum_{v \in V} f_{v}(x(v)) \quad \text { subject to } \quad x \in J \text {, }
$$

where $f_{v}: \mathbb{Z} \rightarrow \mathbb{R}(v \in V)$ is a family of univariate convex functions and $J$ is a finite jump system. The second problem is the minimization of an M-convex function on a constant-parity jump system:

$$
\text { (McFMin) Minimize } f(x) \quad \text { subject to } x \in J \text {, }
$$

where $J \subseteq \mathbb{Z}^{V}$ is a finite constant-parity jump system and $f: J \rightarrow \mathbb{R}$ is an M-convex function. For both of the problems, we assume that a membership oracle for $J$ and an oracle for evaluating the function value of $f$ are available. We present polynomial-time algorithms for the two problems.

\subsection{A Polynomial-Time Algorithm for Minimizing a Separable- Convex Function on a Jump System}

We first show some properties for optimal solutions of the problem (ScFMin). The global optimality of the problem (ScFMin) is characterized by a local optimality.

Theorem 4.1 ([3, Corollary 4.2]). A vector $x \in J$ is an optimal solution of (ScFMin) if and only if $f(x) \leq f(x+s+t)$ for all $s \in N_{1}$ and $t \in N_{1} \cup\{\mathbf{0}\}$ with $x+s+t \in J$.

The next property shows that a given nonoptimal vector in $J$ can be easily separated from an optimal solution. 
Theorem 4.2 (minimizer cut property for (ScFMin)). Let $x \in J$ be a vector which is not an optimal solution of (ScFMin). Suppose that $s_{*} \in N_{1}$ satisfies

$$
\begin{array}{r}
s_{*} \in \arg \min \left\{f(x+s) \mid s \in N_{1}, \exists t \in N_{1} \cup\{\mathbf{0}\}\right. \\
\text { s.t. } x+s+t \in J \text { and } f(x+s+t)<f(x)\} .
\end{array}
$$

Then, there exists an optimal solution $x_{*}$ of (ScFMin) satisfying

$$
\begin{cases}x_{*}(u) \leq x(u)-1 & \left(\text { if } s_{*}=-\chi_{u}\right), \\ x_{*}(u) \geq x(u)+1 & \left(\text { if } s_{*}=+\chi_{u}\right) .\end{cases}
$$

The proof of Theorem 4.2 will be given in Section 4.4.1.

Our algorithm maintains a box $[a, b]$ containing an optimal solution of (ScFMin). Note that $J \cap[a, b]$ is a jump system by Proposition 2.2. The box $[a, b]$ is reduced iteratively by using the minimizer cut property (Theorem 4.2), and finally an optimal solution is found.

Given a finite set $J \subseteq \mathbb{Z}^{V}$, we define a set $J^{\circ} \subseteq \mathbb{Z}^{V}$ by

$$
J^{\circ}=J \cap\left[a_{J}^{\circ}, b_{J}^{\circ}\right],
$$

where

$$
\begin{aligned}
& a_{J}(v)=\min \{y(v) \mid y \in J\}, \quad b_{J}(v)=\max \{y(v) \mid y \in J\} \quad(v \in V), \\
& a_{J}^{\circ}(v)=\left\lfloor\left(1-\frac{1}{n}\right) a_{J}(v)+\frac{1}{n} b_{J}(v)\right\rfloor \quad(v \in V), \\
& b_{J}^{\circ}(v)=\left\lfloor\frac{1}{n} a_{J}(v)+\left(1-\frac{1}{n}\right) b_{J}(v)\right\rceil \quad(v \in V) .
\end{aligned}
$$

The set $J^{\circ}$ is intended to represent a set of vectors in $J$ lying away from the boundary.

Theorem 4.3. Let $J$ be a finite jump system.

(i) $J^{\circ}$ is nonempty and hence a jump system.

(ii) A vector in $J^{\circ}$ can be found in $\mathrm{O}\left(n^{2} \log \Phi(J)\right)$ time, provided a vector in $J$ is given.

Proof. The proof is given in Sections 4.4.2 and 4.4.3.

Algorithm Domain_Reduction_ScFMin

Step 0: Set $a(v):=-\infty$ and $b(v):=+\infty$ for $v \in V$.

Step 1: Find a vector $x \in(J \cap[a, b])^{\circ}$.

Step 2: If $f(x) \leq f(x+s+t)$ for all $s, t \in N_{1} \cup\{\mathbf{0}\}$, then stop ( $x$ is optimal).

Step 3: Find $s_{*} \in N_{1}$ satisfying (4.1).

Step 4: Put $\{u\}=\operatorname{supp}\left(s_{*}\right)$. Modify $a$ or $b$ as follows:

$$
\begin{cases}b(u)=x(u)-1 & \left(\text { if } s_{*}=-\chi_{u}\right), \\ a(u)=x(u)+1 & \left(\text { if } s_{*}=+\chi_{u}\right) .\end{cases}
$$

Go to Step 1.

We analyze the number of iterations of the algorithm. Denote by $a_{i}, b_{i}$ the vectors $a, b$ at the beginning of the $i$-th iteration. It is clear that $b_{i}(v)-a_{i}(v)$ is nonincreasing w.r.t. $i$. Furthermore, we have the following property: 
Lemma 4.4. Let $u \in V$ be the element with $\{u\}=\operatorname{supp}\left(s_{*}\right)$, where $s_{*}$ are the vectors chosen in Step 2 of the $i$-th iteration. Then, we have

$$
b_{i+1}(u)-a_{i+1}(u)<\left(1-\frac{1}{n}\right)\left\{b_{i}(u)-a_{i}(u)\right\} .
$$

Proof. We show the inequality in the case $s_{*}=-\chi_{u}$ only. Let $x \in J \cap\left[a_{i}, b_{i}\right]$ be the vector chosen in Step 1 of the $i$-th iteration. Then,

$$
\begin{aligned}
b_{i+1}(u)-a_{i+1}(u) & =x(u)-1-a_{i}(u) \\
& \leq\left\lceil\frac{1}{n} a_{i}(u)+\left(1-\frac{1}{n}\right) b_{i}(u)\right]-1-a_{i}(u) \\
& <\left(1-\frac{1}{n}\right)\left\{b_{i}(u)-a_{i}(u)\right\} .
\end{aligned}
$$

We have $b_{0}(v)-a_{0}(v) \leq \Phi(J)$ for all $v \in V$ at the beginning of the algorithm, and if $b_{i}(v)-a_{i}(v)<1$ for all $v \in V$, then we obtain an optimal solution immediately. Hence, it follows from Lemma 4.4 that the algorithm DOMAIN_REDUCTION_SCFMin terminates in $\mathrm{O}\left(n^{2} \log \Phi(J)\right)$ iterations.

By Theorem 4.3, a vector in $(J \cap[a, b])^{\circ}$ can be found in $\mathrm{O}\left(n^{2} \log \Phi(J)\right)$ time. Steps 2,3 , and 4 can be done in $\mathrm{O}\left(n^{2}\right)$ time.

Theorem 4.5. The algorithm DoMAIN_REDUCTION_SCFMin finds an optimal solution of the problem (ScFMin) in $\mathrm{O}\left(n^{4}(\log \Phi(J))^{2}\right)$ time, provided a vector in $J$ is given.

\subsection{Application to Weak Majorized Elements in Jump Systems}

We explain an application of our algorithm to the problem of finding least weakly sub- and supermajorized elements in jump systems discussed in [1] (see also [26]).

For a vector $x \in \mathbb{R}^{V}$, let $x_{[1]} \geq x_{[2]} \geq \cdots \geq x_{[n]}$ denote the components of $x$ in decreasing order. For two vectors $x, y \in \mathbb{R}^{V}$, the vector $x$ is said to be weakly submajorized by $y$ if

$$
\sum_{i=1}^{j} x_{[i]} \leq \sum_{i=1}^{j} y_{[i]} \quad(j=1,2, \ldots, n) .
$$

For a nonempty subset $S$ of $\mathbb{R}^{V}$, a vector $x \in S$ is said to be a least weakly submajorized element of $S$ if $x$ is weakly submajorized by $y$ for all $y \in S$.

The concept of weak supermajorization is similarly defined. For a vector $x \in \mathbb{R}^{V}$, let $x_{(1)} \leq x_{(2)} \leq \cdots \leq x_{(n)}$ denote the components of $x$ in increasing order. For two vectors $x, y \in \mathbb{R}^{V}$, the vector $x$ is said to be weakly supermajorized by $y$ if

$$
\sum_{i=1}^{j} x_{(i)} \geq \sum_{i=1}^{j} y_{(i)} \quad(j=1,2, \ldots, n) .
$$


It is easy to see that $x$ is weakly supermajorized by $y$ if and only if $-x$ is weakly submajorized by $-y$. For a nonempty subset $S$ of $\mathbb{R}^{V}$, a vector $x \in S$ is said to be a least weakly supermajorized element of $S$ if $x$ is weakly supermajorized by $y$ for all $y \in S$.

The following statement conjectured by Tamir [26] is proven by Ando [1].

Theorem 4.6 ([1]). Any finite jump system has a least weakly sub-and supermajorized elements.

The proof of Theorem 4.6 in [1] shows that the problem of finding a least weakly submajorized element of a jump system $J$ can be reduced to the following convex quadratic optimization problem:

$$
\text { Minimize } \quad \sum_{v \in V}(x(v)+M)^{2} \quad \text { subject to } \quad x \in J,
$$

where $M$ is a nonnegative real number such that $x(v)+M \geq 0$ for all $x \in J$ and $v \in V$. Such $M$ is given by

$$
M=\left\{\begin{array}{cl}
0 & \left(\text { if } J \subseteq \mathbb{Z}_{+}^{V}\right), \\
-\min _{v \in V}\{\min \{y(v) \mid y \in J\}\} & \text { (otherwise), }
\end{array}\right.
$$

and can be computed in $\mathrm{O}\left(n^{2} \log \Phi(J)\right)$ time by Theorem 3.3. Then, the convex quadratic optimization problem above can be solved in $\mathrm{O}\left(n^{4}(\log \Phi(J))^{2}\right)$ time by using the algorithm Domain_REDUCTION_ScFMin.

Theorem 4.7. Least weakly sub- and supermajorized elements of a finite jump system $J$ can be computed in $\mathrm{O}\left(n^{4}(\log \Phi(J))^{2}\right)$ time, provided a vector in $J$ is given.

\subsection{A Polynomial-Time Algorithm for Minimization of an M-convex Function on a Constant-Parity Jump Sys- tem}

The problem (McFMin) can be solved in polynomial time in a similar way as the problem (ScFMin), due to the following useful properties. The global optimality of the problem (McFMin) is characterized by a local optimality.

Theorem 4.8 ([22, Theorem 3.3]). A vector $x \in J$ is an optimal solution of (McFMin) if and only if $f(x) \leq f(x+s+t)$ holds for all $s \in N_{1}$ and $t \in N_{1}$ with $x+s+t \in J$.

Minimizer cut property holds for the problem (McFMin) as well.

Theorem 4.9 (minimizer cut property for (McFMin) [23, Theorem 4.1]). Let $x \in J$ be a vector which is not an optimal solution of (McFMin), and $s_{*}, t_{*} \in N_{1}$ satisfy

$$
f\left(x+s_{*}+t_{*}\right)=\min \left\{f(x+s+t) \mid s, t \in N_{1}\right\} .
$$

Put $\{u\}=\operatorname{supp}\left(s_{*}\right)$ and $\{w\}=\operatorname{supp}\left(t_{*}\right)$. Then, there exists $x_{*} \in \arg \min f$ such that

$$
x_{*}(u)\left\{\begin{array} { l l } 
{ \leq x ( u ) - 1 } & { ( \text { if } s _ { * } = - \chi _ { u } ) , } \\
{ \geq x ( u ) + 1 } & { ( \text { if } s _ { * } = + \chi _ { u } ) , }
\end{array} \quad x _ { * } ( w ) \left\{\begin{array}{ll}
\leq x(w)-1 & \left(\text { if } t_{*}=-\chi_{w}\right), \\
\geq x(w)+1 & \left(\text { if } t_{*}=+\chi_{w}\right) .
\end{array}\right.\right.
$$


Based on Theorems 4.8 and 4.9, we consider a variant of the algorithm Domain_REDUCTION_ScFMin in Section 4.1.

Algorithm Domain_Reduction_McFMin

Step 0: Set $a(v):=-\infty$ and $b(v):=+\infty$ for $v \in V$.

Step 1: Find a vector $x \in(J \cap[a, b])^{\circ}$.

Step 2: If $f(x) \leq f(x+s+t)$ for all $s, t \in N_{1}$, then stop ( $x$ is optimal).

Step 3: Find $s_{*}, t_{*} \in N_{1}$ satisfying $f\left(x+s_{*}+t_{*}\right)=\min \left\{f(x+s+t) \mid s, t \in N_{1}\right\}$.

Step 4: Put $\{u\}=\operatorname{supp}\left(s_{*}\right)$ and $\{w\}=\operatorname{supp}\left(t_{*}\right)$. Modify $a$ and $b$ as follows:

$\left\{\begin{array}{ll}b(u)=x(u)-1 \quad\left(\text { if } s_{*}=-\chi_{u}\right), \\ a(u)=x(u)+1 \quad\left(\text { if } s_{*}=+\chi_{u}\right),\end{array} \quad \begin{cases}b(w)=x(w)-1 \quad\left(\text { if } t_{*}=-\chi_{w}\right) \\ a(w)=x(w)+1 & \left(\text { if } t_{*}=+\chi_{w}\right)\end{cases}\right.$

Go to Step 1.

We can show the following result, where the proof is quite similar to that for Theorem 4.5 and therefore omitted.

Theorem 4.10. The algorithm DOMAIn_REDUCTION_MCFMin solves the problem (McFMin) in $\mathrm{O}\left(n^{4}(\log \Phi(J))^{2}\right)$ time, provided a vector in $J$ is given.

\subsection{Proofs}

\subsubsection{Proof of Minimizer Cut Property for (ScFMin)}

In this section, we prove Theorem 4.2. A proof of Theorem 4.2 is given for a special case where $J$ is a convex jump system [2, Theorem 5.2]. A jump system $J$ is said to be convex if every integral point in the convex hull of $J$ is contained in $J$. In the following, we give a proof for the general case.

The proof uses the following fundamental properties of separable convex functions.

Proposition 4.11. Let $f: \mathbb{Z}^{V} \rightarrow \mathbb{R}$ be a separable-convex function.

(i) For any $x, y \in \mathbb{Z}^{V}$ and any $s \in \operatorname{inc}(x, y)$, we have

$$
f(x)+f(y) \geq f(x+s)+f(y-s) .
$$

(ii) For any $x \in \mathbb{Z}^{V}$ and distinct $s, t \in N_{1}$, we have

$$
f(x+s+t)-f(x)=\{f(x+s)-f(x)\}+\{f(x+t)-f(x)\} .
$$

Let $x \in J$ be a vector which is not an optimal solution of (ScFMin), and $s_{*} \in N_{1}$ be a vector satisfying (4.1). We assume, without loss of generality, that $s_{*}=+\chi_{u}$ for some $u \in V$. Let $x_{*}$ be an optimal solution of (ScFMin) maximizing the value $x_{*}(u)$, and assume that $x_{*}$ minimizes $\left\|x_{*}-x\right\|_{1}$ among all such $x_{*}$. We assume, to the contrary, that $x_{*}(u) \leq x(u)$ and derive a contradiction.

By the definition of $s_{*}$, there exists $t_{*} \in N_{1} \cup\{\mathbf{0}\}$ such that

$$
x+s_{*}+t_{*} \in J, \quad f\left(x+s_{*}+t_{*}\right)<f(x) .
$$

Lemma 4.12. $f\left(x+s_{*}\right)<f(x)$. 
Proof. We assume $t_{*} \neq \mathbf{0}$ since otherwise the claim is obvious from (4.3). If $t_{*}=s_{*}$, then the separable convexity of $f$ and (4.3) imply $\left\{f\left(x+s_{*}\right)-f(x)\right\} \leq$ $\left\{f\left(x+2 s_{*}\right)-f(x)\right\} / 2<0$. If $t_{*} \neq s_{*}$, then (4.1) and Proposition 4.11 (ii) imply

$2\left\{f\left(x+s_{*}\right)-f(x)\right\} \leq\left\{f\left(x+s_{*}\right)-f(x)\right\}+\left\{f\left(x+t_{*}\right)-f(x)\right\}=f\left(x+s_{*}+t_{*}\right)-f(x)$,

which, together with (4.3), yields $f\left(x+s_{*}\right)<f(x)$.

Lemma 4.13. There exists $p \in \operatorname{inc}\left(x_{*}, x\right)$ such that $f\left(x_{*}+p\right)>f\left(x_{*}\right)$ and $f(x-p)<f\left(x+s_{*}\right)$.

Proof. Since $s_{*} \in \operatorname{inc}\left(x_{*}, x+s_{*}\right)$, Proposition 4.11 (i) and Lemma 4.12 imply

$$
f\left(x_{*}+s_{*}\right)-f\left(x_{*}\right) \leq f\left(x+s_{*}\right)-f(x)<0,
$$

which, together with the optimality of $x_{*}$, yields $x_{*}+s_{*} \notin J$. Since $s_{*} \in$ $\operatorname{inc}\left(x_{*}, x+s_{*}+t_{*}\right),\left(\mathrm{J}-\mathrm{EXC}_{0}\right)$ implies that there exists $p \in \operatorname{inc}\left(x_{*}+s_{*}, x+s_{*}+t_{*}\right)$ such that $x_{*}+s_{*}+p \in J$. By the choice of $x_{*}$, we have

$$
f\left(x_{*}+s_{*}+p\right)>f\left(x_{*}\right) .
$$

Claim 1: $p \neq s_{*}$.

[Proof of Claim] Assume, to the contrary, that $p=s_{*}$. We consider the following two cases and derive a contradiction.

[Case 1: $s_{*}=t_{*}$ ] Separable convexity of $f$, the inequality $x_{*}(u) \leq x(u)$, and (4.3) imply

$$
f\left(x_{*}+2 s_{*}\right)-f\left(x_{*}\right) \leq f\left(x+2 s_{*}\right)-f(x)<0,
$$

which contradicts the inequality (4.5).

[Case $2: s_{*} \neq t_{*}$ ] (4.5) implies $f\left(x_{*}+2 s_{*}\right)>f\left(x_{*}\right)$, from which follows

$$
f\left(x_{*}+2 s_{*}\right)-f\left(x_{*}+s_{*}\right) \geq(1 / 2)\left\{f\left(x_{*}+2 s_{*}\right)-f\left(x_{*}\right)\right\}>0 .
$$

Since $s_{*}=p \in \operatorname{inc}\left(x_{*}+s_{*}, x+s_{*}+t_{*}\right)$, Proposition 4.11 (i) implies

$$
f\left(x+s_{*}+t_{*}\right)-f\left(x+t_{*}\right) \geq f\left(x_{*}+2 s_{*}\right)-f\left(x_{*}+s_{*}\right) .
$$

Since $f\left(x+s_{*}+t_{*}\right)-f\left(x+t_{*}\right)=f\left(x+s_{*}\right)-f(x)$ by Proposition 4.11 (ii), it follows from (4.6) and (4.7) that $f\left(x+s_{*}\right)>f(x)$, a contradiction to Lemma 4.12 .

[End of Claim]

We first show that $p \in \operatorname{inc}\left(x_{*}, x\right)$. Assume, to the contrary, that $p \notin$ $\operatorname{inc}\left(x_{*}, x\right)$. Since $p \in \operatorname{inc}\left(x_{*}+s_{*}, x+s_{*}+t_{*}\right)=\operatorname{inc}\left(x_{*}, x+t_{*}\right)$, we have $p=t_{*}$. Then, $t_{*} \neq s_{*}$ by Claim 1. Therefore, Proposition 4.11 (ii) implies

$$
\begin{aligned}
f\left(x_{*}+s_{*}+p\right)-f\left(x_{*}\right) & =f\left(x_{*}+s_{*}+t_{*}\right)-f\left(x_{*}\right) \\
& =\left\{f\left(x_{*}+s_{*}\right)-f\left(x_{*}\right)\right\}+\left\{f\left(x_{*}+t_{*}\right)-f\left(x_{*}\right)\right\} \\
& \leq\left\{f\left(x+s_{*}\right)-f(x)\right\}+\left\{f\left(x+t_{*}\right)-f(x)\right\} \\
& =f\left(x+s_{*}+t_{*}\right)-f(x)<0,
\end{aligned}
$$

where the first inequality is by $s_{*} \in \operatorname{inc}\left(x_{*}, x+s_{*}\right)$ and $t_{*}=p \in \operatorname{inc}\left(x_{*}, x+t_{*}\right)$, and the second inequality by (4.3). This, however, is a contradiction to (4.5). 
We then show that $f\left(x_{*}+p\right)>f\left(x_{*}\right)$ and $f(x-p)<f\left(x+s_{*}\right)$. By (4.5), Proposition 4.11 (ii), and Claim 1, we have

$$
0<f\left(x_{*}+s_{*}+p\right)-f\left(x_{*}\right)=\left\{f\left(x_{*}+s_{*}\right)-f\left(x_{*}\right)\right\}+\left\{f\left(x_{*}+p\right)-f\left(x_{*}\right)\right\} .
$$

Therefore, it holds that

$$
\begin{aligned}
f(x-p)-f(x) & \leq f\left(x_{*}\right)-f\left(x_{*}+p\right) \\
& <f\left(x_{*}+s_{*}\right)-f\left(x_{*}\right) \\
& \leq f\left(x+s_{*}\right)-f(x)<0,
\end{aligned}
$$

where the first inequality is by Proposition 4.11 (i) and $p \in \operatorname{inc}\left(x_{*}, x\right)$, the second by (4.8), and the last two inequalities are by (4.4). This implies $f\left(x_{*}+p\right)>f\left(x_{*}\right)$ and $f(x-p)<f\left(x+s_{*}\right)$.

Let $p_{1} \in \operatorname{inc}\left(x_{*}, x\right)$ be a vector with $f\left(x_{*}+p_{1}\right)>f\left(x_{*}\right)$ minimizing the value $f\left(x-p_{1}\right)$ among all such vectors. It follows from Lemmas 4.12 and 4.13 that

$$
f\left(x-p_{1}\right)<f\left(x+s_{*}\right)<f(x),
$$

which implies $x-p_{1} \notin J$ by (4.1). Hence, $\left(\mathrm{J}-\mathrm{EXC}_{0}\right)$ implies that there exists $q \in \operatorname{inc}\left(x-p_{1}, x_{*}\right)$ such that $x-p_{1}+q \in J$. By (4.1) and (4.9), we have

$$
f\left(x-p_{1}+q\right) \geq f(x) .
$$

Lemma 4.14. $q \neq-p_{1}$

Proof. Assume, to the contrary, that $q=-p_{1}$. Since $-p_{1}=q \in \operatorname{inc}\left(x-p_{1}, x_{*}\right)$, Proposition 4.11 (i) implies

$$
f\left(x_{*}\right)-f\left(x_{*}+p_{1}\right) \geq f\left(x-2 p_{1}\right)-f\left(x-p_{1}\right) .
$$

By (4.9) and (4.10), we have

$$
f\left(x-2 p_{1}\right)-f\left(x-p_{1}\right) \geq f(x)-f\left(x-p_{1}\right)>0 .
$$

It follows from (4.11) and (4.12) that $f\left(x_{*}\right)>f\left(x_{*}+p_{1}\right)$, a contradiction to the choice of $p_{1}$.

Since $q \in \operatorname{inc}\left(x-p_{1}, x_{*}\right) \subseteq \operatorname{inc}\left(x, x_{*}\right)$, it follows from Proposition 4.11 (i) that

$$
f\left(x_{*}\right)-f\left(x_{*}-q\right) \geq f(x+q)-f(x) .
$$

By Proposition 4.11 (ii), (4.10) and Lemma 4.14, we have

$$
f(x+q)-f(x) \geq f(x)-f\left(x-p_{1}\right) .
$$

It follows from (4.9), (4.13), and (4.14) that

$$
f\left(x_{*}\right)-f\left(x_{*}-q\right) \geq f(x)-f\left(x-p_{1}\right)>0 .
$$

From this inequality we have $x_{*}-q \notin J$ since $x_{*}$ is an optimal solution. Hence, $\left(\mathrm{J}-\mathrm{EXC}_{0}\right)$ implies that there exists $p_{2} \in \operatorname{inc}\left(x_{*}-q, x\right)$ such that $x_{*}-q+p_{2} \in$ $J$. We note that $\left(x_{*}-q+p_{2}\right)(u) \geq x_{*}(u)$ since $-s_{*} \notin\left\{-q, p_{2}\right\}$ and that $\left\|\left(x_{*}-q+p_{2}\right)-x\right\|_{1}<\left\|x_{*}-x\right\|_{1}$. Therefore, we have

$$
f\left(x_{*}-q+p_{2}\right)>f\left(x_{*}\right)
$$

by the choice of $x_{*}$. 
Lemma 4.15. $p_{2} \neq-q$.

Proof. Assume, to the contrary, that $p_{2}=-q$. Since $-q=p_{2} \in \operatorname{inc}\left(x_{*}-q, x\right)$, Proposition 4.11 (i) implies

$$
f(x)-f(x+q) \geq f\left(x_{*}-2 q\right)-f\left(x_{*}-q\right)>0,
$$

where the last inequality is by (4.15) and (4.16). On the other hand, Proposition 4.11 (ii), (4.10), and Lemma 4.14 imply

$$
f(x+q)-f(x) \geq f(x)-f\left(x-p_{1}\right)>0,
$$

where the last inequality is by (4.9). This inequality, however, is a contradiction to $(4.17)$.

By Proposition 4.11 (ii), (4.16) and Lemma 4.15, we have

$$
f\left(x_{*}+p_{2}\right)-f\left(x_{*}\right)>f\left(x_{*}\right)-f\left(x_{*}-q\right) .
$$

Since $p_{2} \in \operatorname{inc}\left(x_{*}-q, x\right) \subseteq \operatorname{inc}\left(x_{*}, x\right)$, it follows from Proposition 4.11 (i) that

$$
f(x)-f\left(x-p_{2}\right) \geq f\left(x_{*}+p_{2}\right)-f\left(x_{*}\right),
$$

which, together with (4.15) and (4.18), implies $f\left(x_{*}+p_{2}\right)>f\left(x_{*}\right)$ and $f(x-$ $\left.p_{2}\right)<f\left(x-p_{1}\right)$, a contradiction to the choice of $p_{1}$.

This concludes the proof of Theorem 4.2.

\subsubsection{Nonemptyness of $J^{\circ}$}

We prove Theorem $4.3(\mathrm{i})$, the nonemptyness of the set $J^{\circ}=J \cap\left[a_{J}^{\circ}, b_{J}^{\circ}\right]$ defined by (4.2).

We first show that the intersection of the convex hull $\operatorname{conv}(J)$ of $J$ and the box $\left[a_{J}^{\circ}, b_{J}^{\circ}\right]$ is nonempty.

We define

$$
3^{V}=\{(X, Y) \mid X, Y \subseteq V, X \cap Y=\emptyset\} .
$$

Given a function $\rho: 3^{V} \rightarrow \mathbb{R}$, we define a polyhedron $P_{*}(\rho)$ as

$$
P_{*}(\rho)=\left\{x \in \mathbb{R}^{V} \mid x(X)-x(Y) \leq \rho(X, Y)\left(\forall(X, Y) \in 3^{V}\right)\right\} .
$$

A function $\rho: 3^{V} \rightarrow \mathbb{R}$ is called a bisubmodular function if it satisfies the following inequality for all $\left(X_{1}, Y_{1}\right),\left(X_{2}, Y_{2}\right) \in 3^{V}$ :

$$
\begin{aligned}
& \rho\left(X_{1}, Y_{1}\right)+\rho\left(X_{2}, Y_{2}\right) \\
& \quad \geq \rho\left(X_{1} \cap X_{2}, Y_{1} \cap Y_{2}\right)+\rho\left(\left(X_{1} \cup X_{2}\right) \backslash\left(Y_{1} \cup Y_{2}\right),\left(Y_{1} \cup Y_{2}\right) \backslash\left(X_{1} \cup X_{2}\right)\right) .
\end{aligned}
$$

Theorem 4.16 ([7]). Let $J \subseteq \mathbb{Z}^{V}$ be a jump system. Then, there exists an integer-valued bisubmodular function $\rho_{J}: 3^{V} \rightarrow \mathbb{Z} \cup\{+\infty\}$ such that $\rho_{J}(\emptyset, \emptyset)=0$ and $\operatorname{conv}(J)=P_{*}\left(\rho_{J}\right)$. Moreover, such $\rho_{J}$ is uniquely determined by

$$
\rho_{J}(X, Y)=\sup \{x(X)-x(Y) \mid x \in J\} \quad\left((X, Y) \in 3^{V}\right) .
$$


Theorem 4.17 ([12]). Let $\rho: 3^{V} \rightarrow \mathbb{R}$ be a bisubmodular function with $\rho(\emptyset, \emptyset)=$ 0 , and $a, b \in \mathbb{R}^{V}$ be vectors with $a \leq b$. Then, the set $P_{*}(\rho) \cap[a, b]$ is nonempty if and only if

$$
a(X)-b(Y) \leq \rho(X, Y) \quad\left(\forall(X, Y) \in 3^{V}\right) .
$$

Lemma 4.18. For a finite jump system $J \subseteq \mathbb{Z}^{V}$, it holds that $\operatorname{conv}(J) \cap$ $\left[a_{J}^{\circ}, b_{J}^{\circ}\right] \neq \emptyset$.

Proof. Let $\rho=\rho_{J}$ be a function defined by (4.19). It follows from Theorem 4.16 that $\rho$ is an integer-valued bisubmodular function satisfying $\rho(\emptyset, \emptyset)=0$ and $\operatorname{conv}(J)=P_{*}\left(\rho_{J}\right)$. Moreover, we have

$$
\begin{array}{ll}
a_{J}^{\circ}(v)=\left\lfloor-\left(1-\frac{1}{n}\right) \rho(\emptyset,\{v\})+\frac{1}{n} \rho(\{v\}, \emptyset)\right] \quad(v \in V), \\
b_{J}^{\circ}(v)=\left[-\frac{1}{n} \rho(\emptyset,\{v\})+\left(1-\frac{1}{n}\right) \rho(\{v\}, \emptyset)\right] \quad(v \in V)
\end{array}
$$

since $\rho(\emptyset,\{v\})=-a_{J}(v)$ and $\rho(\{v\}, \emptyset)=b_{J}(v)$ hold. To prove $\operatorname{conv}(J) \cap$ $\left[a_{J}^{\circ}, b_{J}^{\circ}\right] \neq \emptyset$, it suffices to show that $a_{J}^{\circ}(X)-b_{J}^{\circ}(Y) \leq \rho(X, Y)$ for all $(X, Y) \in 3^{V}$ by Theorem 4.17 .

Let $(X, Y) \in 3^{V}$ and put $k=|X|+|Y|$. We claim that

$$
\begin{aligned}
& k \rho(X, Y)+k \sum_{v \in Y} \rho(\{v\}, \emptyset)+k \sum_{v \in X} \rho(\emptyset,\{v\}) \\
& \geq \sum_{v \in Y}\{\rho(\{v\}, \emptyset)+\rho(\emptyset,\{v\})\}+\sum_{v \in X}\{\rho(\{v\}, \emptyset)+\rho(\emptyset,\{v\})\} .
\end{aligned}
$$

Indeed, the bisubmodularity of $\rho$ implies

$$
\begin{aligned}
\text { LHS of (4.23) }= & \sum_{w \in Y}\left\{\rho(X, Y)+\sum_{v \in Y \backslash\{w\}} \rho(\{v\}, \emptyset)+\sum_{v \in X} \rho(\emptyset,\{v\})\right\} \\
& +\sum_{w \in X}\left\{\rho(X, Y)+\sum_{v \in Y} \rho(\{v\}, \emptyset)+\sum_{v \in X \backslash\{w\}} \rho(\emptyset,\{v\})\right\} \\
& +\sum_{v \in Y} \rho(\{v\}, \emptyset)+\sum_{v \in X} \rho(\emptyset,\{v\}) \\
\geq & \sum_{w \in Y}\{\rho(X, Y)+\rho(Y \backslash\{w\}, \emptyset)+\rho(\emptyset, X)\} \\
& +\sum_{w \in X}\{\rho(X, Y)+\rho(Y, \emptyset)+\rho(\emptyset, X \backslash\{w\})\} \\
& +\sum_{v \in Y} \rho(\{v\}, \emptyset)+\sum_{v \in X} \rho(\emptyset,\{v\}) \\
\geq & \operatorname{RHS} \text { of }(4.23) .
\end{aligned}
$$

Since the LHS of (4.23) is nonnegative and $k \leq n$, the integer $k$ in (4.23) can 
be replaced with $n$. Thus,

$$
\begin{aligned}
\rho(X, Y) \geq & \sum_{v \in X}\left\{-\left(1-\frac{1}{n}\right) \rho(\emptyset,\{v\})+\frac{1}{n} \rho(\{v\}, \emptyset)\right\} \\
& -\sum_{v \in Y}\left\{-\frac{1}{n} \rho(\emptyset,\{v\})+\left(1-\frac{1}{n}\right) \rho(\{v\}, \emptyset)\right\} \\
\geq & a_{J}^{\circ}(X)-b_{J}^{\circ}(Y),
\end{aligned}
$$

where the last inequality follows from (4.21) and (4.22).

We prove the nonemptyness of $J^{\circ}$ by using the following theorem.

Theorem 4.19 ([18, Theorem 5.1]). Let $J$ be a finite jump system and $a, b \in$ $\mathbb{Z}^{V}$ be vectors with $a(v)<b(v)$ for all $v \in V$. Then, there exists a vector $w \in\{-1,0,+1\}^{V}$ such that

$\min \left\{\|x-y\|_{1} \mid x \in[a, b], y \in J\right\}=\min \left\{w^{T} x \mid x \in[a, b]\right\}-\max \left\{w^{T} y \mid y \in J\right\}$.

Lemma 4.20. For a finite jump system $J$, the set $J^{\circ}$ defined by (4.2) is nonempty.

Proof. Let $V^{\prime}=\left\{v \in V \mid a_{J}^{\circ}(v)<b_{J}^{\circ}(v)\right\}$. We denote by $J^{\prime} \subseteq \mathbb{Z}^{V^{\prime}}$ the orthogonal projection of $J$ onto $\mathbb{Z}^{V^{\prime}}$, i.e.,

$$
J^{\prime}=\left\{y \in \mathbb{Z}^{V^{\prime}} \mid \exists x \in J \text { such that } y(v)=x(v)\left(v \in V^{\prime}\right)\right\} .
$$

For $v \in V \backslash V^{\prime}$, we have $a_{J}^{\circ}(v)=b_{J}^{\circ}(v)=a_{J}(v)=b_{J}(v)$, implying that $y(v)=$ $a_{J}^{\circ}(v)\left(=b_{J}^{\circ}(v)\right)$ for all $y \in J$. Therefore, $J \cap\left[a_{J}^{\circ}, b_{J}^{\circ}\right] \neq \emptyset$ if and only if

$$
J^{\prime} \cap\left\{x \in \mathbb{Z}^{V^{\prime}} \mid a_{J}^{\circ}(v) \leq x(v) \leq b_{J}^{\circ}(v)\left(v \in V^{\prime}\right)\right\} \neq \emptyset,
$$

where it is noted that $a_{J^{\prime}}^{\circ}(v)=a_{J}^{\circ}(v)$ and $b_{J^{\prime}}^{\circ}(v)=b_{J}^{\circ}(v)$ for $v \in V^{\prime}$. Hence, it suffices to consider the case where $a_{J}^{\circ}(v)<b_{J}^{\circ}(v)$ for all $v \in V$.

By Theorem 4.19, there exists some $w \in\{-1,0,+1\}^{V}$ such that

$$
\begin{aligned}
& \min \left\{\|x-y\|_{1} \mid x \in\left[a_{J}^{\circ}, b_{J}^{\circ}\right], y \in J\right\} \\
& \quad=\min \left\{w^{T} x \mid x \in\left[a_{J}^{\circ}, b_{J}^{\circ}\right]\right\}-\max \left\{w^{T} y \mid y \in J\right\} .
\end{aligned}
$$

Since $\operatorname{conv}(J) \cap\left[a_{J}^{\circ}, b_{J}^{\circ}\right] \neq \emptyset$ by Lemma 4.18 , we have

$$
\begin{aligned}
& \min \left\{w^{T} x \mid x \in\left[a_{J}^{\circ}, b_{J}^{\circ}\right]\right\}-\max \left\{w^{T} y \mid y \in J\right\} \\
& \quad=\min \left\{w^{T} x \mid x \in\left[a_{J}^{\circ}, b_{J}^{\circ}\right]\right\}-\max \left\{w^{T} y \mid y \in \operatorname{conv}(J)\right\} \leq 0 .
\end{aligned}
$$

It follows from (4.24) and (4.25) that $\min \left\{\|x-y\|_{1} \mid x \in\left[a_{J}^{\circ}, b_{J}^{\circ}\right], y \in J\right\}=0$, implying $J^{\circ}=J \cap\left[a_{J}^{\circ}, b_{J}^{\circ}\right] \neq \emptyset$.

This concludes the proof of Theorem 4.3 (i). 


\subsubsection{Finding a Vector in $J^{\circ}$}

We prove Theorem 4.3 (ii) by providing an algorithm to find a vector in $J^{\circ}$. More generally, we consider how to find a vector in the (nonempty) intersection of a jump system $J$ and a box $[a, b]$.

Our algorithm is based on the following simple observation.

Lemma 4.21. Let $J$ be a jump system, $u \in V$, and $\alpha, \beta$ be integers such that $\alpha \leq \beta$ and $J \cap\left\{y \in \mathbb{Z}^{V} \mid \alpha \leq y(u) \leq \beta\right\} \neq \emptyset$. Then, we have

$$
\begin{aligned}
& \max \{y(u) \mid y \in J, y(u) \leq \beta\} \geq \alpha, \\
& \min \{y(u) \mid y \in J, y(u) \geq \alpha\} \leq \beta .
\end{aligned}
$$

Proof. Let $x$ be any vector in $J \cap\left\{y \in \mathbb{Z}^{V} \mid \alpha \leq y(u) \leq \beta\right\}$. Then, we have

$$
\begin{aligned}
& \max \{y(u) \mid y \in J, y(u) \leq \beta\} \geq x(u) \geq \alpha, \\
& \min \{y(u) \mid y \in J, y(u) \geq \alpha\} \leq x(u) \leq \beta .
\end{aligned}
$$

Given a jump system $J$ and vectors $a, b \in \mathbb{Z}^{V}$ with $a \leq b$ and $J \cap[a, b] \neq \emptyset$, the following algorithm finds a vector in $J \cap[a, b]$, provided a vector in $J$ is given.

\section{Algorithm Find_VeCtor_IN_ $J \cap[a, b]$}

Step 0: Let $x:=x_{0}$ be an initial vector in $J$.

Step 1: For each $u \in V$ with $x(u)<a(u)$, do the following:

Step 1-1: Find a vector $x_{*}$ in $J^{\prime}$ maximizing the value $x_{*}(u)$, where

$$
\begin{aligned}
J^{\prime}= & J \cap\left\{y \in \mathbb{Z}^{V} \mid y(u) \leq b(u),\right. \\
& \min (x(v), a(v)) \leq y(v) \leq \max (x(v), b(v))(v \in V \backslash\{u\})\} .
\end{aligned}
$$

Step 1-2: Put $x:=x_{*}$.

Step 2: For each $u \in V$ with $x(u)>b(u)$, do the following:

Step 2-1: Find a vector $x_{*}$ in $J^{\prime}$ minimizing the value $x_{*}(u)$, where

$$
\begin{aligned}
J^{\prime}= & J \cap\left\{y \in \mathbb{Z}^{V} \mid y(u) \geq a(u),\right. \\
& \min (x(v), a(v)) \leq y(v) \leq \max (x(v), b(v))(v \in V \backslash\{u\})\} .
\end{aligned}
$$

Step 2-2: Put $x:=x_{*}$

Step 3: Output $x$.

We observe that if the inequality $a(v) \leq x(v) \leq b(v)$ for some $v \in V$ is once satisfied, then it is kept until termination of the algorithm. Note that the set $J^{\prime}$ defined in Step 1-1 is a jump system by Proposition 2.2. This, together with Lemma 4.21, implies that the vector $x$ satisfies $x \in J^{\prime}$ and $a(u) \leq x(u) \leq b(u)$ just immediately after Step 1-2. Similarly, for each $u \in V$ with $x(u)>b(u)$, the inequality $a(u) \leq x(u) \leq b(u)$ is satisfied just immediately after Step 2-2. Thus, the vector $x$ satisfies $x \in J \cap[a, b]$ at the end of the algorithm.

Each iteration of Steps 1 and Step 2 requires $\mathrm{O}\left(n \log \Phi\left(J^{\prime}\right)\right)$ time by Corollary 3.4 , and we have $\Phi\left(J^{\prime}\right) \leq \Phi(J)$ since $J^{\prime} \subseteq J$. Hence, the algorithm runs in $\mathrm{O}\left(n^{2} \log \Phi(J)\right)$ time.

This concludes the proof of Theorem 4.3 (ii). 


\section{Acknowledgements}

The first author is supported by the Humboldt Research Fellowship of the Alexander von Humboldt Foundation. The second author is supported by the Research Fellowship of the Japan Society for the Promotion of Science for Young Scientists.

\section{References}

[1] K. Ando, Weak majorization of finite jump systems, manuscript (1996). Available from http://coconut.sys.eng.shizuoka.ac.jp/ando/maj/maj11.dvi

[2] K. Ando, S. Fujishige, and T. Naitoh, A greedy algorithm for minimizing a separable convex function over an integral bisubmodular polyhedron, J. Oper. Res. Soc. Japan, 37 (1994), pp. 188-196.

[3] K. Ando, S. Fujishige, and T. Naitoh, A greedy algorithm for minimizing a separable convex function over a finite jump system, J. Oper. Res. Soc. Japan, 38 (1995), pp. 362-375.

[4] N. Apollonio and A. Sebö, Minsquare factors and maxfix covers of a graphs, in Integer Programming and Combinatorial Optimization, D. Bienstock, G. Nemhauser, eds., Lecture Notes in Computer Science 3064, Springer, Berlin, 2004, pp. 388-400.

[5] N. Apollonio and A. Sebö, Minconvex factors of prescribed size in graphs, preprint No. 145, Leibniz-IMAG (2006).

[6] A. Bouchet, Greedy algorithm and symmetric matroids, Math. Programming, 38 (1987), pp. 147-159.

[7] A. Bouchet and W. H. Cunningham, Delta-matroids, jump systems and bisubmodular polyhedra, SIAM J. Discrete Math., 8 (1995), pp. 17-32.

[8] R. Chandrasekaran and S. N. Kabadi, Pseudomatroids, Discrete Math., 71 (1988), pp. 205-217.

[9] W. H. Cunningham, Matching, matroids, and extensions, Math. Programming, 91 (2002), pp. 515-542.

[10] S. Fujishige, A min-max theorem for bisubmodular polyhedra, SIAM J. Discrete Math., 10 (1997), pp. 294-308.

[11] S. Fujishige, Submodular Functions and Optimization, 2nd ed., Annals of Discrete Mathematics, 58, Elsevier, Amsterdam, 2005.

[12] S. Fujishige and S. B. Patkar, The box convolution and the Dilworth truncation of bisubmodular functions, Report No. 94823, Forschungsinstitut für Diskrete Mathematik, Universität Bonn (1994).

[13] J. F. Geelen, Lectures on jump system, manuscript (1996). Available from http://www.math. uwaterloo.ca/ ${ }^{\sim}$ jfgeelen/publications/js.ps 
[14] H. Groenevelt, Two algorithms for maximizing a separable concave function over a polymatroid feasible region, Euro. J. Oper. Res., 54 (1991), pp. 227236.

[15] D. S. Hochbaum, Lower and upper bounds for the allocation problem and other nonlinear optimization problems, Math. Oper. Res., 19 (1994), pp. 390-409.

[16] Y. Kobayashi, K. Murota, and K. Tanaka: Operations on M-convex functions on jump systems, METR 2006-13, Department of Mathematical Informatics, University of Tokyo (2006).

[17] E. L. Lawler, Combinatorial Optimization: Networks and Matroids, Holt, Rinehart and Winston, New York, NY, 1976, Dover Publications, Mineola, NY, 2001.

[18] L. Lovász, The membership problem in jump systems, J. Combin. Theory, Ser. B, 70 (1997), pp. 45-66.

[19] A. W. Marshall and I. Olkin, Inequalities: Theory of Majorization and its Applications, Academic Press, New York, NY, 1979.

[20] S. Moriguchi and A. Shioura, On Hochbaum's scaling algorithm for the general resource allocation problem, Math. Oper. Res., 29 (2004), pp. 394397.

[21] K. Murota, Discrete Convex Analysis, SIAM, Philadelphia, PA, 2003.

[22] K. Murota, M-convex functions on jump systems: a general framework for minsquare factor problem, SIAM J. Discrete Math., 20 (2006), 213-226.

[23] K. Murota and K. Tanaka, A steepest descent algorithm for M-convex functions on jump systems, IEICE Trans. Fundamentals, E89-A (2006), to appear.

[24] A. Schrijver, Combinatorial Optimization: Polyhedra and Efficiency, Springer, Berlin, 2003.

[25] A. Shioura, Minimization of an M-convex function, Discrete Appl. Math., 84 (1998), pp. 215-220.

[26] A. Tamir, Least majorized elements and generalized polymatroids, Math. Oper. Res., 20 (1995), pp. 583-589.

[27] D. Welsh, Matroid Theory, Academic Press, New York, NY, 1976. 